\title{
A strong convergence theorem on solving common solutions for generalized equilibrium problems and fixed-point problems in Banach space
}

De-ning $\mathrm{Qu}^{1,2}$ and Cao-zong Cheng ${ }^{1 *}$

\footnotetext{
* Correspondence: czcheng@bjut. edu.cn

${ }^{1}$ College of Applied Science, Beijing University of Technology, Beijing 100124, PR China Full list of author information is available at the end of the article
}

\begin{abstract}
In this paper, the common solution problem (P1) of generalized equilibrium problems for a system of inverse-strongly monotone mappings $\left\{A_{k}\right\}_{k=1}^{N}$ and a system of bifunctions $\left\{f_{k}\right\}_{k=1}^{N}$ satisfying certain conditions, and the common fixed-point problem (P2) for a family of uniformly quasi- $\varphi$-asymptotically nonexpansive and locally uniformly Lipschitz continuous or uniformly Hölder continuous mappings $\left\{S_{i}\right\}_{i=1}^{\infty}$ are proposed. A new iterative sequence is constructed by using the generalized projection and hybrid method, and a strong convergence theorem is proved on approximating a common solution of (P1) and (P2) in Banach space. 2000 MSC: 26B25, 40A05

Keywords: Common solution, Equilibrium problem, Fixed-point problem, Iterative sequence, Strong convergence
\end{abstract}

\section{Introduction}

Recently, common solution problems (i.e., to find a common element of the set of solutions of equilibrium problems and/or the set of fixed points of mappings and/or the set of solutions of variational inequalities) with their applications have been discussed. Some authors such as in references [1-7] presented various iterative schemes and showed some strong or weak convergence theorems on common solution problems in Hilbert spaces. In 2008-2009, Takahashi and Zembayashi [8,9] introduced several iterative sequences on finding a common solution of an equilibrium problem and a fixed-point problem for a relatively nonexpansive mapping, and established some strong or weak convergence theorems. In 2010, Chang et al. [10] discussed the common solution of a generalized equilibrium problem and a common fixed-point problem for two relatively nonexpansive mappings, and established a strong convergence theorem on the common solution problem. The frameworks of spaces in [8-10] are the uniformly smooth and uniformly convex Banach spaces. Chang et al. [11] established a strong convergence theorem on solving the common fixed-point problem for a family of uniformly quasi- $\varphi$-asymptotically nonexpansive and uniformly Lipschitz continuous mappings in a uniformly smooth and strictly convex Banach space with the KadecKlee property. Some other problems such as optimization problems (e.g. see $[1,4,6]$ )

\section{SpringerOpen ${ }^{\circ}$}

(c) 2011 Qu and Cheng; licensee Springer. This is an Open Access article distributed under the terms of the Creative Commons Attribution License (http://creativecommons.org/licenses/by/2.0), which permits unrestricted use, distribution, and reproduction in any medium, provided the original work is properly cited. 
and common zero-point problems (e.g. see [10]) are closely related to common solution problems.

Throughout this paper, unless other stated, $\mathbb{R}$ and $\mathbb{l}$ are denoted by the set of the real numbers and the set $\{1,2, \ldots, N\}$, respectively, where $N$ is any given positive integer. Let $E$ be a real Banach space with the norm $\|\cdot\|, E^{*}$ be the dual of $E$, and $\langle\cdot, \cdot\rangle$ be the pairing between $E$ and $E^{*}$. Suppose that $C$ is a nonempty closed convex subset of $E$.

Let $\left\{A_{k}\right\}_{k=1}^{N}: C \rightarrow E^{*}$ be $N$ mappings and $\left\{f_{k}\right\}_{k=1}^{N}: C \times C \rightarrow \mathbb{R}$ be $N$ bifunctions. For each $k \in \mathbb{V}$, the generalized equilibrium problem for $f_{k}$ and $A_{k}$ is to seek $\bar{u} \in C$ such that

$$
f_{k}(\bar{u}, y)+\left\langle y-\bar{u}, A_{k} \bar{u}\right\} \geq 0, \quad \forall y \in C .
$$

The common solution problem (P1) of generalized equilibrium problems for $\left\{A_{k}\right\}_{k=1}^{N}$ and $\left\{f_{k}\right\}_{k=1}^{N}$ is to seek an element in $\mathbb{G}$, where $\mathbb{G}=\bigcap_{k=1}^{N} G(k)$ and $G(k)$ is the set of solutions of (1.1). We write $G$ instead of $\mathbb{G}$ in the case of $N=1$.

Let $\left\{S_{i}\right\}_{i=1}^{\infty}: C \rightarrow C$ be a family of mappings. The common fixed-point problem (P2) for $\left\{S_{i}\right\}_{i=1}^{\infty}$ is to seek an element in $\mathbb{F}$, where $\mathbb{E}=\bigcap_{i=1}^{\infty} F\left(S_{i}\right)$ and $F\left(S_{i}\right)$ is the set of fixed points of $S_{i}$.

Motivated by the works in [8-11], in this paper we will produce a new iterative sequence approximating a common solution of (P1) and (P2) (i.e., some point belonging to $\mathbb{F} \cap \mathbb{G}$ ), and show a strong convergence theorem in a uniformly smooth and strictly convex Banach space with the Kadec-Klee property, where $\left\{S_{i}\right\}_{i=1}^{\infty}$ in (P2) is a family of uniformly quasi- $\varphi$-asymptotically nonexpansive mappings and for each $i \geq 1$, $S_{i}$ is locally uniformly Lipschitz continuous or uniformly Hölder continuous with order $\Theta_{i}$.

\section{Preliminaries}

Let $E$ be a real Banach space, and $\left\{x_{n}\right\}$ be a sequence in $E$. We denote by $x_{n} \rightarrow x$ and $x_{n} \rightarrow x$ the strong convergence and weak convergence of $\left\{x_{n}\right\}$, respectively. The normalized duality mapping $J: E \rightarrow 2^{E^{*}}$ is defined by

$$
J x=\left\{f \in E^{*}:\langle x, f\rangle=\|x\|^{2}=\|f\|^{2}\right\}, \quad \forall x \in E .
$$

By the Hahn-Banach theorem, $J x \neq \varnothing$ for each $x \in E$.

A Banach space $E$ is said to be strictly convex if $\frac{\|x+y\|}{2}<1$ for all $x, y \in U=\{u \in E$ $:\|u\|=1\}$ with $x \neq y$; to be uniformly convex if for each $\varepsilon \in(0,2]$, there exists $\gamma>0$ such that $\frac{\|x+y\|}{2}<1-\gamma$ for all $x, y \in U$ with $\|x-y\| \geq \varepsilon$; to be smooth if the limit

$$
\lim _{t \rightarrow 0} \frac{\|x+t y\|-\|x\|}{t}
$$

exists for every $x, y \in U$; to be uniformly smooth if the limit (2.1) exists uniformly for all $x, y \in U$.

Remark 2.1. The basic properties below hold (see [12]).

(i) If $E$ is a real uniformly smooth Banach space, then $J$ is uniformly continuous on each bounded subset of $E$.

(ii) If $E$ is a strictly convex reflexive Banach space, then $J^{1}$ is hemicontinuous, that is, $J^{1}$ is norm-to-weak*-continuous. 
(iii) If $E$ is a smooth and strictly convex reflexive Banach space, then $J$ is singlevalued, one-to-one and onto.

(iv) Each uniformly convex Banach space $E$ has the Kadec-Klee property, that is, for any sequence $\left\{x_{n}\right\} \subset E$, if $x_{n} \rightarrow x \in E$ and $\left\|x_{n}\right\| \rightarrow\|x\|$, then $x_{n} \rightarrow x$.

(v) A Banach space $E$ is uniformly smooth if and only if $E^{*}$ is uniformly convex.

(vi) A Banach space $E$ is strictly convex if and only if $J$ is strictly monotone, that is,

$$
\left\langle x-y, x^{*}-y^{*}\right\rangle>0 \quad \text { whenever } x, y \in E, \quad x \neq y \text { and } x^{*} \in J x, y^{*} \in J y .
$$

(vii) Both uniformly smooth Banach spaces and uniformly convex Banach spaces are reflexive.

Now let $E$ be a smooth and strictly convex reflexive Banach space. As Alber [13] and Kamimura and Takahashi [14] did, the Lyapunov functional $\varphi: E \times E \rightarrow \mathbb{R}^{+}$is defined by

$$
\phi(x, y)=\|x\|^{2}-2\langle x, J y\rangle+\|y\|^{2}, \quad \forall x, y \in E .
$$

It follows from [15] that $\varphi(x, y)=0$ if and only if $x=y$, and that

$$
(\|x\|-\|y\|)^{2} \leq \phi(x, y) \leq(\|x\|+\|y\|)^{2} .
$$

Further suppose that $C$ is a nonempty closed convex subset of $E$. The generalized projection (see [13]) $\Pi_{C}: E \rightarrow C$ is defined by for each $x \in E$,

$$
\Pi_{C}(x)=\arg \min _{y \in C} \phi(y, x) .
$$

A mapping $A: C \rightarrow E^{*}$ is said to be $\delta$-inverse-strongly monotone, if there exists a constant $\delta>0$ such that

$$
\langle x-y, A x-A y\rangle \geq \delta\|A x-A y\|^{2}, \quad \forall x, y \in C .
$$

A mapping $S: C \rightarrow C$ is said to be closed if for each $\left\{x_{n}\right\} \subset C, x_{n} \rightarrow x$ and $S x_{n} \rightarrow y$ imply $S x=y$; to be quasi- $\varphi$-asymptotically nonexpansive (see [16]) if $F(S) \neq \varnothing$, and there exists a sequence $\left\{l_{n}\right\} \subset[1, \infty)$ with $l_{n} \rightarrow 1$ such that

$$
\phi\left(u, S^{n} x\right) \leq l_{n} \phi(u, x), \quad \forall x \in C, u \in F(S), \quad \forall n \geq 1 .
$$

It is easy to see that if $A: C \rightarrow E^{*}$ is $\delta$-inverse-strongly monotone, then $A$ is $\frac{1}{\delta}$-Lipschitz continuous. The class of quasi- $\varphi$-asymptotically nonexpansive mappings contains properly the class of relatively nonexpansive mappings (see [17]) as a subclass.

Definition 2.1 (see [11]). Let $\left\{S_{i}\right\}_{i=1}^{\infty}: C \rightarrow C$ be a sequence of mappings. $\left\{S_{i}\right\}_{i=1}^{\infty}$ is said to be a family of uniformly quasi- $\varphi$-asymptotically nonexpansive mappings, if $\mathbb{F} \neq \emptyset$ and there exists a sequence $\left\{l_{n}\right\} \subset[1, \infty)$ with $l_{n} \rightarrow 1$ such that for each $i \geq 1$,

$$
\phi\left(u, S_{i}^{n} x\right) \leq l_{n} \phi(u, x), \quad \forall u \in \mathbb{F}, x \in C, \quad \forall n \geq 1 .
$$

Now we introduce the following concepts.

Definition 2.2. A mapping $S: C \rightarrow C$ is said

(1) to be locally uniformly Lipschitz continuous if for any bounded subset $D$ in $C$, there exists a constant $L_{D}>0$ such that

$$
\left\|S^{n} x-S^{n} y\right\| \leq L_{D}\|x-y\|, \quad \forall x, y \in D, \quad \forall n \geq 1 ;
$$


(2) to be uniformly Hölder continuous with order $\Theta(\Theta>0)$ if there exists a constant $L>0$ such that

$$
\left\|S^{n} x-S^{n} y\right\| \leq L\|x-y\|^{\Theta}, \quad \forall x, y \in C, \quad \forall n \geq 1 .
$$

Remark 2.2. It is easy to see that any uniformly Lipschitz continuous mapping (see [11]) is locally uniformly Lipschitz continuous, and is also uniformly Hölder continuous with order $\Theta=1$. However, the converse is not true.

Example 2.1. Suppose that $S: \mathbb{R} \rightarrow \mathbb{R}$ is defined by

$$
S(x)=\left\{\begin{aligned}
x^{2}, \text { if } x<0 \\
0, \text { if } x \geq 0
\end{aligned}\right.
$$

Then $S$ is locally uniformly Lipschitz continuous. In fact, for any bounded subset $D$ in $\mathbb{R}$, setting $M=1+\sup \{|x|: x \in D\}$, we have $\left|S^{n} x-S^{n} y\right| \leq 2 M|x-y|, x, y \in D, \forall n$ $\geq 1$. But $S$ fails to be uniformly Lipschitz continuous.

Example 2.2. Suppose that $S: \mathbb{R}-\mathbb{R}$ is defined by

$$
S(x)=\left\{\begin{array}{cc}
\sqrt{-x}, & \text { if } x<0 \\
0, & \text { if } x \geq 0 .
\end{array}\right.
$$

$S$ is uniformly Hölder continuous with order $\Theta=\frac{1}{2}$, since $\left|S^{n} x-S^{n} y\right| \leq 2|x-y|^{\frac{1}{2}}, \forall x$, $y \in \mathbb{R}, \forall n \geq 1$. But $S$ fails to be uniformly Lipschitz continuous.

Lemma 2.1 (see [13,14]). If $C$ is a nonempty closed convex subset of a smooth and strictly convex reflexive Banach space $E$, then

(1) $\varphi\left(x, \Pi_{C}(y)\right)+\varphi\left(\Pi_{C}(y), y\right) \geq \varphi(x, y), \forall x \in C, y \in E$;

(2) for $x \in E$ and $u \in C$, one has

$$
u=\Pi_{C}(x) \Leftrightarrow\langle u-y, J x-J u\rangle \geq 0, \quad \forall y \in C .
$$

Lemma 2.2. Let $E$ be a uniformly smooth and strictly convex Banach space with the Kadec-Klee property, $\left\{x_{n}\right\}$ and $\left\{y_{n}\right\}$ be two sequences of $E$, and $\bar{u} \in E$. If $x_{n} \rightarrow \bar{u}$ and $\varphi\left(x_{n}\right.$, $\left.y_{n}\right) \rightarrow 0$, then $y_{n} \rightarrow \bar{u}$.

Proof. We complete this proof by two steps.

Step 1. Show that there exists a subsequence $\left\{y_{n_{k}}\right\}$ of $\left\{y_{n}\right\}$ such that $y_{n_{k}} \rightarrow \bar{u}$.

In fact, since $\varphi\left(x_{n}, y_{n}\right) \rightarrow 0$, by (2.2) we have $\left\|x_{n}\right\|-\left\|y_{n}\right\| \rightarrow 0$. It follows from $x_{n} \rightarrow \bar{u}$ that

$$
\left\|y_{n}\right\| \rightarrow\|\bar{u}\|(\text { as } n \rightarrow \infty),
$$

and so

$$
\left\|J y_{n}\right\| \rightarrow\|\bar{u}\|(\text { as } n \rightarrow \infty) .
$$

Then $\left\{y_{n}\right\}$ is bounded in $E^{*}$. It follows from Remark 2.1(v) and (vii) that $E^{*}$ is reflexive. Hence there exist a point $f_{0} \in E^{*}$ and a subsequence $\left\{J y_{n_{k}}\right\}$ of $\left\{y y_{n}\right\}$ such that

$$
J y_{n_{k}} \rightarrow f_{0}(\text { as } k \rightarrow \infty) .
$$

It follows from Remark 2.1(vii) and (iii) that there exists a point $x \in E$ such that $J x=$ $f_{0}$. By the definition of $\varphi$, we obtain 


$$
\begin{aligned}
\phi\left(x_{n_{k}}, y_{n_{k}}\right) & =\left\|x_{n_{k}}\right\|^{2}-2\left\langle x_{n_{k}}, J y_{n_{k}}\right\rangle+\left\|y_{n_{k}}\right\|^{2} \\
& =\left\|x_{n_{k}}\right\|^{2}-2\left\langle x_{n_{k}}, J y_{n_{k}}\right\rangle+\left\|J y_{n_{k}}\right\|^{2} .
\end{aligned}
$$

By weak lower semicontinuity of norm $\|\cdot\|$, we have

$$
\begin{aligned}
0 & =\liminf _{k \rightarrow \infty} \phi\left(x_{n_{k}}, y_{n_{k}}\right) \\
& \geq\|\bar{u}\|^{2}-2\left\langle\bar{u}, f_{0}\right\rangle+\left\|f_{0}\right\|^{2} \\
& =\|\bar{u}\|^{2}-2\langle\bar{u}, J x\rangle+\|J x\|^{2} \\
& =\|\bar{u}\|^{2}-2\langle\bar{u}, J x\rangle+\|x\|^{2}=\phi(\bar{u}, x),
\end{aligned}
$$

which implies that $\bar{u}=x$ and $f_{0}=J \bar{u}$. It follows from Remark 2.1(iv) and (v) that $E^{* *}$ has the Kadec-Klee property, and so $J y_{n_{k}} \rightarrow J \bar{u}$ by (2.4) and (2.5). By Remark 2.1(vii) and (ii), we have $y_{n_{k}} \rightarrow \bar{u}$, which implies that $y_{n_{k}} \rightarrow \bar{u}$ by (2.3) and the Kadec-Klee property of $E$.

Step 2. Show that $y_{n} \rightarrow \bar{u}$.

In fact, suppose that $y_{n} \nrightarrow \rightarrow \bar{u}$. For some given number $\varepsilon_{0}>0$, there exists a positive integer sequence $\left\{n_{k}\right\}$ with $n_{1}<n_{2}<\cdots<n_{k}<\cdots$, such that

$$
\left\|\gamma_{n_{k}}-\bar{u}\right\| \geq \varepsilon_{0} .
$$

Replacing $\left\{y_{n}\right\}$ by $\left\{y_{n_{k}}\right\}$ in Step 1 , there exists a subsequence $\left\{y_{n_{k_{k}}}\right\}$ of $\left\{y_{n_{k}}\right\}$ such that $y_{n_{k_{i}}} \rightarrow \bar{u}$, which contradicts (2.6).

Lemma 2.3. Let $C$ be a nonempty closed convex subset of a smooth and strictly convex reflexive Banach space $E$, and let $A: C \rightarrow E^{*}$ be a $\delta$-inverse-strongly monotone mapping and $f: C \times C \rightarrow \mathbb{R}$ be a bifunction satisfying the following conditions

$\left(\mathrm{B}_{1}\right) f(z, z)=0, \forall z \in C$;

$\left(\mathrm{B}_{2}\right) \limsup _{t \downarrow 0} f(z+t(x-z), y) \leq f(z, y), \quad \forall x, y, \quad z \in C$;

$\left(\mathrm{B}_{3}\right)$ for any $z \in C$, the function $y \propto f(z, y)$ is convex and lower semicontinuous;

$\left(\mathrm{B}_{4}\right)$ for some $\beta \geq 0$ with $\beta \leq \delta$,

$$
f(z, y)+f(y, z) \leq \beta\|A z-A y\|^{2}, \quad \forall z, y \in C .
$$

Then the following conclusions hold:

(1) For any $r>0$ and $u \in E$, there exists a unique point $z \in C$ such that

$$
f(z, y)+\langle y-z, A z\rangle+\frac{1}{r}\langle y-z, J z-J u\rangle \geq 0, \quad \forall y \in C .
$$

(2) For any given $r>0$, define a mapping $K_{r}: E \rightarrow C$ as follows: $\forall u \in E$,

$$
K_{r} u=z \text { such that } f(z, y)+\langle y-z, A z\rangle+\frac{1}{r}\langle y-z, J z-J u\rangle \geq 0, \quad \forall y \in C .
$$

We have (i) $F\left(K_{r}\right)=G$ and $G$ is closed convex in $C$, where

$$
G=\{z \in C: f(z, y)+\langle y-z, A z\rangle \geq 0, \quad \forall y \in C\} ;
$$

(ii) $\varphi\left(z, K_{r} u\right)+\varphi\left(K_{r} u, u\right) \leq \varphi(z, u), \forall z \in F\left(K_{r}\right)$.

(3) For each $n \geq 1, r_{n}>a>0$ and $u_{n} \in C$ with $\lim _{n \rightarrow \infty} u_{n}=\lim _{n \rightarrow \infty} K_{r_{n}} u_{n}=\bar{u}$, we have

$$
f(\bar{u}, y)+\langle y-\bar{u}, A \bar{u}\rangle \geq 0, \quad \forall y \in C .
$$


Proof. (1) We consider the bifunction $\tilde{f}:(z, y) \mapsto f(z, y)+\langle y-z, A z\rangle$ instead of $f$. It follows from the proof of Lemma 2.5 in [10] that $\tilde{f}$ satisfies $\left(B_{1}\right)-\left(B_{3}\right)$. Since $A$ is $\delta$ inverse-strongly monotone, by $\left(\mathrm{B}_{4}\right)$, we have

$$
\begin{aligned}
& (f(z, y)+\langle y-z, A z\rangle)+(f(y, z)+\langle z-y, A y\rangle) \\
= & f(z, y)+f(y, z)-\langle z-y, A z-A y\rangle \\
\leq & (\beta-\delta)\|A z-A y\|^{2} \leq 0, \forall y, z \in C,
\end{aligned}
$$

which implies $\tilde{f}$ is monotone. By Blum amd Oettli [18], for any $r>0$ and $u \in E$, there exists $z \in C$ such that (2.7) holds. Next we show that (2.7) has a unique solution. If for any given $r>0$ and $u \in E, z_{1}$ and $z_{2}$ are two solutions of (2.7), then

$$
f\left(z_{1}, z_{2}\right)+\left\langle z_{2}-z_{1}, A z_{1}\right\rangle+\frac{1}{r}\left\langle z_{2}-z_{1}, J z_{1}-J u\right\rangle \geq 0,
$$

and

$$
f\left(z_{2}, z_{1}\right)+\left\langle z_{1}-z_{2}, A z_{2}\right\rangle+\frac{1}{r}\left\langle z_{1}-z_{2}, J z_{2}-J u\right\rangle \geq 0 .
$$

Adding these two inequalities, we have

$$
f\left(z_{1}, z_{2}\right)+f\left(z_{2}, z_{1}\right)-\left\langle z_{2}-z_{1}, A z_{2}-A z_{1}\right\rangle-\frac{1}{r}\left\langle z_{2}-z_{1}, J z_{2}-J z_{1}\right\rangle \geq 0 .
$$

It follows from (2.8) that

$$
\left\langle z_{2}-z_{1}, J z_{2}-J z_{1}\right\rangle \leq 0,
$$

which implies that $z_{1}=z_{2}$ by Remark 2.1(vi).

(2) Since $\tilde{f}$ satisfies $\left(B_{1}\right)-\left(B_{3}\right)$ and is monotone, the conclusion (2) follows from Lemmas 2.8 and 2.9 in [9].

(3) Since

$$
f\left(K_{r_{n}} u_{n}, y\right)+\left\langle y-K_{r_{n}} u_{n}, A K_{r_{n}} u_{n}\right\rangle+\frac{1}{r_{n}}\left\langle y-K_{r_{n}} u_{n}, J K_{r_{n}} u_{n}-J u_{n}\right\rangle \geq 0, \quad \forall y \in C,
$$

we have

$$
\begin{aligned}
\frac{1}{r_{n}}\left\langle y-K_{r_{n}} u_{n}, J K_{r_{n}} u_{n}-J u_{n}\right\rangle & \geq-\left(f\left(K_{r_{n}} u_{n}, y\right)+\left\langle y-K_{r_{n}} u_{n}, A K_{r_{n}} u_{n}\right\rangle\right) \\
& \geq f\left(y, K_{r_{n}} u_{n}\right)+\left\langle K_{r_{n}} u_{n}-y, A y\right\rangle, \quad \forall y \in C,
\end{aligned}
$$

by the monotonicity of $\tilde{f}$. It follows from $\lim _{n \rightarrow \infty} u_{n}=\lim _{n \rightarrow \infty} K_{r_{n}} u_{n}=\bar{u} . r_{n}>a>0$ and Remark 2.1(i) that

$$
\lim _{n \rightarrow \infty} \frac{\left\|J u_{n}-J K_{r_{n}} u_{n}\right\|}{r_{n}}=0 .
$$

Since $y \mapsto \tilde{f}(z, y)$ is convex and lower semicontinuous, it is also weakly lower semicontinuous. Letting $n \rightarrow \infty$ in (2.9), we have $f(y, \bar{u})+\langle\bar{u}-y, A y\rangle \leq 0, \forall y \in C$. For any $t \in(0,1]$ and $y \in C$, setting $y_{t}=t y+(1-t) \bar{u}$, we have $y_{t} \in C$ and $f\left(y_{t}, \bar{u}\right)+\left\langle\bar{u}-y_{t}, A y_{t}\right\rangle \leq 0$, which together with $\left(\mathrm{B}_{1}\right)$ implies that 


$$
\begin{aligned}
0 & =f\left(y_{t}, y_{t}\right)+\left\langle y_{t}-y_{t}, A y_{t}\right\rangle \\
& =f\left(y_{t}, t y+(1-t) \bar{u}\right)+\left\langle t y+(1-t) \bar{u}-y_{t}, A y_{t}\right\rangle \\
& \leq t\left[f\left(y_{t}, y\right)+\left\langle y-y_{t}, A y_{t}\right\rangle\right]+(1-t)\left[f\left(y_{t}, \bar{u}\right)+\left\langle\bar{u}-y_{t}, A y_{t}\right\rangle\right] \\
& \leq t\left[f\left(y_{t}, y\right)+\left\langle y-y_{t}, A y_{t}\right\rangle\right] .
\end{aligned}
$$

Thus $f\left(y_{t}, y\right)+\left\langle y-y_{t}, A y_{t}\right\rangle \geq 0, \forall y \in C, \forall t \in(0,1]$. Letting $t \downarrow 0$, since $z \alpha f(z, y)+\langle y$ - $z$, Az satisfies $\left(\mathrm{B}_{2}\right)$, we have $f(\bar{u}, y)+\langle y-\bar{u}, A \bar{u}\rangle \geq 0, \forall y \in C$.

Remark 2.3. If $\beta=0$ in $\left(\mathrm{B}_{4}\right)$, that is, $f$ is monotone, then the conclusions (1) and (2) in Lemma 2.3 reduce to the relating results of Lemmas 2.5 and 2.6 in [10], respectively.

Next we give an example to show that there exist the mapping $A$ and the bifunction $f$ satisfying the conditions of Lemma 2.3. However, $f$ is not monotone.

Example 2.3. Define $A: \mathbb{R} \rightarrow \mathbb{R}$ and $f: \mathbb{R} \times \mathbb{R} \rightarrow \mathbb{R}$ by $A x=2 x+\sqrt{1+x^{2}} \in \forall x \in \mathbb{R}$ and $f(x, y)=\frac{(x-y)^{2}}{10}, \forall(x, y) \in \mathbb{R} \times \mathbb{R}$, respectively. It is easy to see that $A$ is $\frac{1}{3}$-inversestrongly monotone, $f$ satisfies $\left(\mathrm{B}_{1}\right)-\left(\mathrm{B}_{3}\right)$, and $f(x, y)+f(y, x) \leq \frac{1}{5}|A x-A y|^{2}, \forall(x, y): \mathbb{R}$ $\times \mathbb{R}$ with $\frac{1}{5} \leq \frac{1}{3}$.

Lemma 2.4 (see [12]). Let $C$ be a nonempty closed convex subset of a real uniformly smooth and strictly convex Banach space E with the Kadec-Klee property, $S: C \rightarrow C$ be a closed and quasi- $\varphi$-asymptotically nonexpansive mapping with a sequence $\left\{l_{n}\right\} \subset[1$, $\infty), l_{n} \rightarrow 1$. Then $F(S)$ is closed convex in $C$.

Lemma 2.5 (see [11]). Let $E$ be a uniformly convex Banach space, $\eta>0$ be a positive number and $B_{\eta}(0)$ be a closed ball of $E$. Then, for any given sequence $\left\{x_{n}\right\}_{n=1}^{\infty} \subset B_{\eta}(0)$ and for any given $\left\{\lambda_{n}\right\}_{n=1}^{\infty} \subset(0,1)$ with $\sum_{n=1}^{\infty} \lambda_{n}=1$, there exists a continuous, strictly increasing and convex function $g:[0,2 \eta) \rightarrow[0, \infty)$ with $g(0)=0$ such that for any positive integers $i, j$ with $i<j$,

$$
\left\|\sum_{n=1}^{\infty} \lambda_{n} x_{n}\right\|^{2} \leq \sum_{n=1}^{\infty} \lambda_{n}\left\|x_{n}\right\|^{2}-\lambda_{i} \lambda_{j} g\left(\left\|x_{i}-x_{j}\right\|\right) .
$$

\section{Strong convergence theorem}

In this section, let $C$ be a nonempty closed convex subset of a real uniformly smooth and strictly convex Banach space $E$ with the Kadec-Klee property.

Theorem 3.1. Suppose that

$\left(\mathrm{C}_{1}\right)$ for each $k \in \mathbb{J}$, the mapping $A_{k}: C \rightarrow E^{*}$ is $\delta_{k}$-inverse-strongly monotone, the bifunction $f_{k}: C \times C \rightarrow \mathbb{R}$ satisfies $\left(\mathrm{B}_{1}\right)-\left(\mathrm{B}_{3}\right)$, and for some $\beta_{k} \geq 0$ with $\beta_{k} \leq \delta_{k}$,

$$
f_{k}(z, y)+f_{k}(y, z) \leq \beta_{k}\left\|A_{k} z-A_{k} y\right\|, \quad \forall z, y \in C ;
$$

$\left(C_{2}\right)\left\{S_{i}\right\}_{i=1}^{\infty}: C \rightarrow$ Cis a family of closed and uniformly quasi- $\varphi$-asymptotically nonexpansive mappings with a sequence $\left\{l_{n}\right\} \subset[1, \infty), l_{n} \rightarrow 1$;

$\left(C_{3}\right)$ for each $i \geq 1, S_{i}$ is either locally uniformly Lipschitz continuous or uniformly Hölder continuous with order $\Theta_{i}\left(\Theta_{i}>0\right)$, and $\mathbb{F}$ is bounded in $C$. 
$\left(\mathrm{C}_{4}\right) \mathbb{E} \cap \mathbb{G} \neq \emptyset$. Take the sequence $\left\{x_{n}\right\}_{n=1}^{\infty}$ generated by

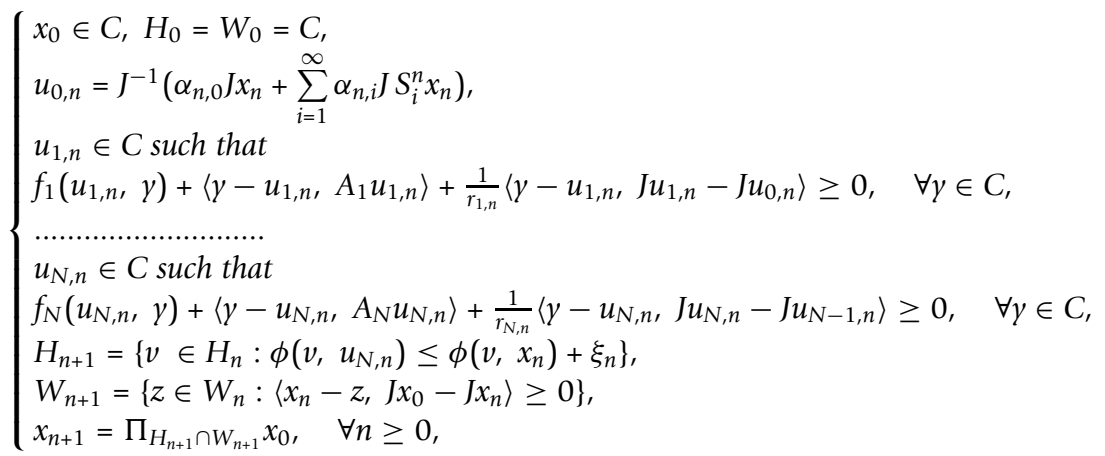

where for each $k \in \mathbb{J},\left\{r_{k, n}\right\}_{n=0}^{\infty} \subset[a, \infty)$ with some $a>0,\left\{\alpha_{n, i}\right\}_{n=0, i=0}^{\infty} \subset[0,1]$, and $\xi_{n}=\sup _{u \in \mathbb{F}}\left(l_{n}-1\right) \phi\left(u, x_{n}\right)$. If $\sum_{i=0}^{\infty} \alpha_{n, i}=1, \forall n \geq 0$ and $\lim _{\inf _{n \rightarrow \infty}} \alpha_{n, 0} \alpha_{n, i}>0, \forall i \geq 1$, then $x_{n} \rightarrow \Pi_{\mathbb{F} \cap \mathbb{G}} x_{0}$.

Proof. We shall complete this proof by seven steps below.

Step 1. Show that $\mathbb{E}, \mathbb{G}, H_{n}$ and $W_{n}$ for all $n \geq 0$ are closed convex.

In fact, $\mathbb{F}=\bigcap_{i=1}^{\infty} F\left(S_{i}\right)$ is closed convex since for each $i \geq 1, F\left(S_{i}\right)$ is closed convex by $\left(C_{2}\right)$ and Lemma 2.4. $\mathbb{G}$ is closed convex since for each $k \in \sqrt{ }, G(k)$ is closed convex by $\left(C_{1}\right)$ and Lemma 2.3(2)(i). $H_{0}=C$ is closed convex. Since $\varphi\left(v, u_{N, n}\right) \leq \varphi\left(v, x_{n}\right)+\xi_{n}$ is equivalent to

$$
2\left\langle v, J x_{n}-J u_{N, n}\right\rangle \leq\left\|x_{n}\right\|^{2}-\left\|u_{N, n}\right\|^{2}+\xi_{n},
$$

we know that $H_{n}(n \geq 0)$ are closed convex. Finally, $W_{n}$ is closed convex by its definition. Thus $\Pi_{\mathbb{F} \cap \mathbb{G}} x_{0}$ and $\Pi_{H_{n} \cap W_{n}} x_{0}$ are well defined.

Step 2. Show that $\left\{x_{n}\right\}$ and $\left\{S_{i}^{n} x_{n}\right\}_{i, n=1}^{\infty}$ are bounded.

From $x_{n}=\Pi_{H_{n} \cap W_{n}} x_{0}, \forall n \geq 0$ and Lemma 2.1(1), we have

$$
\phi\left(x_{n}, x_{0}\right) \leq \phi\left(u, x_{0}\right)-\phi\left(u, x_{n}\right) \leq \phi\left(u, x_{0}\right), \quad \forall u \in C, \quad \forall n \geq 0,
$$

which implies that $\left\{\varphi\left(x_{n}, x_{0}\right)\right\}$ is bounded, and so is $\left\{x_{n}\right\}$ by (2.2). It follows from $\left(C_{2}\right)$ that for all $u \in \mathbb{F}, i \geq 1, n \geq 1$,

$$
\phi\left(u, S_{i}^{n} x_{n}\right) \leq l_{n} \phi\left(u, x_{n}\right) \leq l_{n}\left(\|u\|+\left\|x_{n}\right\|\right)^{2} \leq \sup _{u \in \mathbb{F}} l_{n}\left(\|u\|+\left\|x_{n}\right\|\right)^{2} .
$$

Hence for all $i \geq 1,\left\{\phi\left(u, S_{i}^{n} x_{n}\right)\right\}_{n=1}^{\infty}$ is uniformly bounded, and so is $\left\{S_{i}^{n} x_{n}\right\}_{n=1}^{\infty}$ by (2.2). Obviously,

$$
\xi_{n}=\sup _{u \in \mathbb{F}}\left(l_{n}-1\right) \phi\left(u, x_{n}\right) \leq \sup _{u \in \mathbb{F}}\left(l_{n}-1\right)\left(\|u\|+\left\|x_{n}\right\|\right)^{2} \rightarrow 0(\text { as } n \rightarrow \infty) .
$$

Step 3. Show that $\mathbb{F} \cap \mathbb{G} \subset H_{n} \cap W_{n}, \forall n \geq 0$.

Since Banach space $E$ is uniformly smooth, $E^{*}$ is uniformly convex, by Remark 2.1(v). For any given $p \in \mathbb{F}$, any $n \geq 1$ and any positive integer $j$, by $\left(C_{2}\right)$ and Lemma 2.5 , we have 


$$
\begin{aligned}
\phi\left(p, u_{0, n}\right)= & \phi\left(p, J^{-1}\left(\alpha_{n, 0} J x_{n}+\sum_{i=1}^{\infty} \alpha_{n, i} J S_{i}^{n} x_{n}\right)\right) \\
= & \|p\|^{2}-2\left\langle p, \alpha_{n, 0} J x_{n}+\sum_{i=1}^{\infty} \alpha_{n, i} J S_{i}^{n} x_{n}\right\rangle+\left\|\alpha_{n, 0} J x_{n}+\sum_{i=1}^{\infty} \alpha_{n, i} J S_{i}^{n} x_{n}\right\|^{2} \\
\leq & \|p\|^{2}-2 \alpha_{n, 0}\left\langle p, J x_{n}\right\rangle-2 \sum_{i=1}^{\infty} \alpha_{n, i}\left\langle p, J S_{i}^{n} x_{n}\right\rangle+\alpha_{n, 0}\left\|x_{n}\right\|^{2} \\
& \quad+\sum_{i=1}^{\infty} \alpha_{n, i}\left\|S_{i}^{n} x_{n}\right\|^{2}-\alpha_{n, 0} \alpha_{n, j} g\left(\left\|J x_{n}-J S_{j}^{n} x_{n}\right\|\right) \quad(\text { By Lemma 2.5) } \\
= & \alpha_{n, 0} \phi\left(p, x_{n}\right)+\left(1-\alpha_{n, 0}\right)\|p\|^{2}-2 \sum_{i=1}^{\infty} \alpha_{n, i}\left\langle p, J S_{i}^{n} x_{n}\right\rangle \\
& \quad+\sum_{i=1}^{\infty} \alpha_{n, i}\left\|S_{i}^{n} x_{n}\right\|^{2}-\alpha_{n, 0} \alpha_{n, j} g\left(\left\|J x_{n}-J S_{j}^{n} x_{n}\right\|\right) \\
= & \alpha_{n, 0} \phi\left(p, x_{n}\right)+\sum_{i=1}^{\infty} \alpha_{n, i} \phi\left(p, S_{i}^{n} x_{n}\right)-\alpha_{n, 0} \alpha_{n, j} g\left(\left\|J x_{n}-J S_{j}^{n} x_{n}\right\|\right) \\
\leq & \alpha_{n, 0} \phi\left(p, x_{n}\right)+\sum_{i=1}^{\infty} \alpha_{n, i} l_{n} \phi\left(p, x_{n}\right)-\alpha_{n, 0} \alpha_{n, j} g\left(\left\|J x_{n}-J S_{j}^{n} x_{n}\right\|\right) \\
\leq & l_{n} \phi\left(p, x_{n}\right)-\alpha_{n, 0} \alpha_{n, j} g\left(\left\|J x_{n}-J S_{j}^{n} x_{n}\right\|\right) \\
\leq & \phi\left(p, x_{n}\right)+\sup _{p \in \mathbb{F}}\left(l_{n}-1\right) \phi\left(p, x_{n}\right)-\alpha_{n, 0} \alpha_{n, j} g\left(\left\|J x_{n}-J S_{j}^{n} x_{n}\right\|\right) \\
= & \left.x_{n}\right)+\xi_{n}-\alpha_{n, 0} \alpha_{n, j} g\left(\left\|J x_{n}-J S_{j}^{n} x_{n}\right\|\right) .
\end{aligned}
$$

Put $u_{k, n}=K_{r_{k, n}} u_{k-1, n}, k \in \mathbb{J}, \forall n \geq 0$. It follows from (3.3) and Lemma 2.3(2)(ii) that

$$
\begin{aligned}
\phi\left(p, u_{k, n}\right)=\phi\left(p, K_{r_{k, n}} u_{k-1, n}\right) \leq & \phi\left(p, u_{k-1, n}\right) \leq \phi\left(p, x_{n}\right)+\xi_{n} \\
& \forall p \in \mathbb{F} \cap \mathbb{G}, \quad \forall k \in \mathbb{J}, \quad \forall n \geq 0,
\end{aligned}
$$

which implies that if $p \in \mathbb{E} \cap \mathbb{G}$, then $p \in H_{n}, \forall n \geq 0$. Hence, $\mathbb{F} \cap \mathbb{G} \subset H_{n}, \forall n \geq 0$. By induction, now we prove that $\mathbb{F} \cap \mathbb{G} \subset W_{n}, \forall n \geq 0$. In fact, it follows from $W_{0}=C$ that $\mathbb{F} \cap \mathbb{G} \subset W_{0}$. Suppose that $\mathbb{F} \cap \mathbb{G} \subset W_{m}$ for some $m \geq 0$. By the definition of $x_{m}=\Pi_{H_{m} \cap W_{m}} x_{0}$ and Lemma 2.1(2), we have

$$
\left\langle x_{m}-z, J x_{0}-J x_{m}\right\rangle \geq 0, \quad \forall z \in H_{m} \cap W_{m},
$$

and so

$$
\left\langle x_{m}-z, J x_{0}-J x_{m}\right\rangle \geq 0, \quad \forall z \in \mathbb{F} \cap \mathbb{G},
$$

which shows $z \in W_{m+1}$, so $\mathbb{E} \cap \mathbb{G} \subset W_{m+1}$.

Step 4. Show that there exists $\bar{u} \in C$ such that $x_{n} \rightarrow \bar{u}$.

Without loss of generalization, we can assume that $x_{n} \rightarrow \bar{u}$, since $\left\{x_{n}\right\}$ is bounded and $E$ is reflexive. Moreover, it follows that $\bar{u} \in H_{n} \cap W_{n}, \forall n \geq 0$ from $H_{n+1} \cap W_{n+1} \subset H_{n} \cap$ $W_{n}$ and the closeness and convexity of $H_{n} \cap W_{n}$. Noting that

$$
\begin{aligned}
& \liminf _{n \rightarrow \infty} \phi\left(x_{n}, x_{0}\right)=\liminf _{n \rightarrow \infty}\left(\left\|x_{n}\right\|^{2}-2\left\langle x_{n}, J x_{0}\right\rangle+\left\|x_{0}\right\|^{2}\right) \\
\geq & \|\bar{u}\|^{2}-2\left\langle\bar{u}, J x_{0}\right\rangle+\left\|x_{0}\right\|^{2}=\phi\left(\bar{u}, x_{0}\right),
\end{aligned}
$$


we have

$$
\phi\left(\bar{u}, x_{0}\right) \leq \liminf _{n \rightarrow \infty} \phi\left(x_{n}, x_{0}\right) \leq \limsup _{n \rightarrow \infty} \phi\left(x_{n}, x_{0}\right) \leq \phi\left(\bar{u}, x_{0}\right) .
$$

by (3.1). It follows that

$$
\lim _{n \rightarrow \infty} \phi\left(x_{n}, x_{0}\right)=\phi\left(\bar{u}, x_{0}\right)
$$

and so $\left\|x_{n}\right\| \rightarrow\|\bar{u}\|$ by $x_{n} \rightarrow \bar{u}$. Hence,

$$
x_{n} \rightarrow \bar{u}(\text { as } n \rightarrow \infty)
$$

by the Kadec-Klee property of $E$, and so

$$
J x_{n} \rightarrow J \bar{u}(\text { as } n \rightarrow \infty)
$$

by Remark 2.1(i).

Step 5. Show that $\bar{u} \in \mathbb{E}$.

Since $x_{n+1} \in C$, setting $u=x_{n+1}$ in (3.1), we have

$$
\phi\left(x_{n+1}, x_{n}\right) \leq \phi\left(x_{n+1}, x_{0}\right)-\phi\left(x_{n}, x_{0}\right) .
$$

By (3.5),

$$
\phi\left(x_{n+1}, x_{n}\right) \rightarrow 0(\text { as } n \rightarrow \infty) .
$$

By $x_{n+1} \in H_{n+1}$, (3.2) and (3.8), we have

$$
\phi\left(x_{n+1}, u_{N, n}\right) \leq \phi\left(x_{n+1}, x_{n}\right)+\xi_{n} \rightarrow 0(\text { as } n \rightarrow \infty),
$$

which together with (3.6) and Lemma 2.2 implies that

$$
\lim _{n \rightarrow \infty} u_{N, n}=\bar{u} .
$$

For any $j \geq 1$ and any given $p \in \mathbb{E} \cap \mathbb{G}$, it follows from (3.2)-(3.4) and (3.9) that

$$
\begin{aligned}
& \alpha_{n, 0} \alpha_{n, j} g\left(\left\|J x_{n}-J S_{j}^{n} x_{n}\right\|\right) \leq \phi\left(p, x_{n}\right)+\xi_{n}-\phi\left(p, u_{0, n}\right) \\
\leq & \phi\left(p, x_{n}\right)+\xi_{n}-\phi\left(p, u_{N, n}\right) \rightarrow 0(\text { as } n \rightarrow \infty),
\end{aligned}
$$

which implies that

$$
g\left(|| J x_{n}-J S_{j}^{n} x_{n} \|\right) \rightarrow 0(\text { as } n \rightarrow \infty),
$$

since $\liminf _{n \rightarrow 0} \alpha_{n, 0} \alpha_{n, i}>0, \forall i \geq 1$. We obtain

$$
\left\|J x_{n}-J S_{j}^{n} x_{n}\right\| \rightarrow 0(\text { as } n \rightarrow \infty),
$$

since $g(0)=0$ and $g$ is strictly increasing and continuous. By (3.7) and (3.11), we have $J S_{j}^{n} x_{n} \rightarrow J \bar{u}$ and $\left\|S_{j}^{n} x_{n}\right\| \rightarrow\|\bar{u}\|$ for all $j \geq 1$. It follows from Remark 2.1(ii) that $S_{j}^{n} x_{n} \rightarrow \bar{u}$, which implies

$$
S_{j}^{n} x_{n} \rightarrow \bar{u}(\text { as } n \rightarrow \infty), \quad \forall j \geq 1,
$$

by the uniform boundedness of $\left\{S_{j}^{n} x_{n}\right\}_{n=1}^{\infty}$ and the Kadec-Klee property of $E$. Thus

$$
\left\|S_{j}^{n+1} x_{n+1}-S_{j}^{n} x_{n}\right\| \rightarrow 0(\text { as } n \rightarrow \infty), \quad \forall j \geq 1 .
$$


By $\left(\mathrm{C}_{3}\right)$ and (3.6), we have

$$
\left\|S_{j}^{n+1} x_{n}-S_{j}^{n+1} x_{n+1}\right\| \rightarrow 0(\text { as } n \rightarrow \infty), \quad \forall j \geq 1 .
$$

Hence, for each $j \geq 1$,

$$
\begin{aligned}
& \left\|S_{j}\left(S_{j}^{n} x_{n}\right)-S_{j}^{n} x_{n}\right\|=\left\|S_{j}^{n+1} x_{n}-S_{j}^{n} x_{n}\right\| \\
\leq & \left\|S_{j}^{n+1} x_{n}-S_{j}^{n+1} x_{n+1}\right\|+\left\|S_{j}^{n+1} x_{n+1}-S_{j}^{n} x_{n}\right\| \rightarrow 0(\text { as } n \rightarrow \infty) .
\end{aligned}
$$

By (3.12) and the closeness of $S_{j}$, we have $S_{j} \bar{u}=\bar{u}$ for all $j \geq 1$ and so $\bar{u} \in \mathbb{F}$.

Step 6. Show that $\bar{u} \in \mathbb{G}$.

In fact, it is easy to see that for each $k \in\{0\} \cup \sqrt{ }$, and $p \in \mathbb{F} \cap \mathbb{G}$, the sequence $\{\varphi(p$, $\left.\left.u_{k, n}\right)\right\}$ is bounded by (3.2), (3.4) and the boundedness of $\left\{x_{n}\right\}$ and $\mathbb{F}$, which implies that $\left\{u_{k, n}\right\}$ is bounded in $C$ by (2.2). Since $\bar{u} \in \mathbb{F}$, by (3.2), (3.3), (3.5) and (3.10), we have

$$
\begin{aligned}
& \left.\phi\left(\bar{u}, u_{0, n}\right) \leq \phi\left(\bar{u}, x_{n}\right)+\xi_{n}-\alpha_{n, 0} \alpha_{n, j} g\left(\| J x_{n}-J S_{j}^{n} x_{n}\right) \|\right) \\
\leq & \phi\left(\bar{u}, x_{n}\right)+\xi_{n} \rightarrow 0(\text { as } n \rightarrow \infty) .
\end{aligned}
$$

It follows from Lemma 2.2 that

$$
u_{0, n} \rightarrow \bar{u}(\text { as } n \rightarrow \infty) .
$$

Furthermore, it follows from (3.4) and Lemma 2.3(2)(ii) that for any given $p \in \mathbb{F} \cap \mathbb{G}$,

$$
\phi\left(p, u_{N, n}\right)+\phi\left(u_{1, n}, u_{0, n}\right) \leq \phi\left(p, u_{1, n}\right)+\phi\left(u_{1, n}, u_{0, n}\right) \leq \phi\left(p, u_{0, n}\right),
$$

which implies

$$
\begin{aligned}
& \phi\left(u_{1, n}, u_{0, n}\right) \leq \phi\left(p, u_{0, n}\right)-\phi\left(p, u_{N, n}\right) \\
= & \left\|u_{0, n}\right\|^{2}-\left\|u_{N, n}\right\|^{2}-2\left\langle p, J u_{0, n}-J u_{N, n}\right\rangle \rightarrow 0(\text { as } n \rightarrow \infty),
\end{aligned}
$$

by Remark 2.1(i), (3.9) and (3.13). Then $u_{1, n} \rightarrow \bar{u}$ by (3.13) and Lemma 2.2. Similarly, we also obtain $u_{k, n} \rightarrow \bar{u}(k=2,3, \ldots, N-1)$. Hence, together with (3.9) and (3.13), for each $k \in\{0\} \cup \sqrt{ }$,

$$
u_{k, n} \rightarrow \bar{u}(\text { as } n \rightarrow \infty) \text {. }
$$

For each $k \in \sqrt{ }$, since $u_{k, n}=K_{r_{k, n}} u_{k-1, n}$, we have

$$
f_{k}\left(u_{k, n}, \gamma\right)+\left\langle y-u_{k, n}, A_{k} u_{k, n}\right\rangle+\frac{1}{r_{k, n}}\left\langle y-u_{k, n} J u_{k, n}-J u_{k-1, n}\right\rangle \geq 0, \quad \forall y \in C,
$$

which together with (3.14) and Lemma 2.3(3) implies that $f_{k}(\bar{u}, y)+\left\langle y-\bar{u}, A_{k} \bar{u}\right\rangle \geq 0$, $\forall y \in C$. Therefore $\bar{u} \in \mathbb{G}$ and so $\bar{u} \in \mathbb{F} \cap \mathbb{G}$.

Step 7. Show that $\bar{u}=\Pi_{\mathbb{E} \cap \mathbb{G}} x_{0}$.

In fact, letting $w=\Pi_{\mathbb{F} \cap \mathbb{G} x_{0}}$, by $w \in \mathbb{F} \cap \mathbb{G} \subset H_{n} \cap W_{n}$ and $x_{n}=\Pi_{H_{n} \cap W_{n}} x_{0}$, we have

$$
\phi\left(x_{n}, x_{0}\right) \leq \phi\left(w, x_{0}\right), \quad \forall n \geq 0 .
$$

It follows from (3.6) that

$$
\begin{aligned}
& \phi\left(\bar{u}, x_{0}\right)=\|\bar{u}\|^{2}-2\left\langle\bar{u}, J x_{0}\right\}+\left\|x_{0}\right\|^{2} \\
= & \lim _{n \rightarrow \infty}\left\{\left\|x_{n}\right\|^{2}-2\left\langle x_{n}, J x_{0}\right\}+\left\|x_{0}\right\|^{2}\right\} \\
= & \lim _{n \rightarrow \infty} \phi\left(x_{n}, x_{0}\right) \leq \phi\left(w, x_{0}\right) .
\end{aligned}
$$


Hence, $\bar{u}=w$, and so $x_{n} \rightarrow \bar{u}=\prod_{\mathbb{F} \cap \mathbb{G}} x_{0}$. $\square$

Setting $N=1, u_{0, n}=y_{n}$ and $u_{N, n}=u_{n}$ in Theorem 3.1, we can obtain the following result.

\section{Corollary 3.1 Suppose that}

$\left(\mathrm{D}_{1}\right)$ the mapping $A: C \rightarrow E^{*}$ is a mapping with $\delta$-inverse-strongly monotone, the bifunction $f: \mathrm{C} \times \mathrm{C} \rightarrow \mathbb{R}$ satisfies $\left(\mathrm{B}_{1}\right)-\left(\mathrm{B}_{3}\right)$ and for some $\beta>0$ with $\beta \leq \delta$,

$$
f(z, y)+f(y, z) \leq \beta\|A z-A y\|^{2}, \quad \forall z, y \in C ;
$$

$\left(D_{2}\right)$ both $\left(C_{2}\right)$ and $\left(C_{3}\right)$ hold, and $\mathbb{E} \cap \mathbb{G} \neq \emptyset$ Take the sequence $\left\{x_{n}\right\}_{n=1}^{\infty}$ generated by

$$
\left\{\begin{array}{l}
x_{0} \in C, H_{0}=W_{0}=C, \\
y_{n}=J^{-1}\left(\alpha_{n, 0} J x_{n}+\sum_{i=1}^{\infty} \alpha_{n, i} J S_{i}^{n} x_{n}\right), \\
\quad u_{n} \in C \text { such that } \\
\quad f\left(u_{n}, y\right)+\left\langle y-u_{n}, A u_{n}\right\rangle+\frac{1}{r_{n}}\left\langle y-u_{n}, J u_{n}-J y_{n}\right\} \geq 0, \quad \forall y \in C, \\
H_{n+1}=\left\{v \in H_{n}: \phi\left(v, u_{n}\right) \leq \phi\left(v, x_{n}\right)+\xi_{n}\right\}, \\
W_{n+1}=\left\{z \in W_{n}:\left\langle x_{n}-z, J x_{0}-J x_{n}\right\rangle \geq 0\right\}, \\
x_{n+1}=\Pi_{H_{n+1} \cap W_{n+1}} x_{0}, \quad \forall n \geq 0,
\end{array}\right.
$$

where $\left\{\alpha_{n, i}\right\}_{n=0, i=0}^{\infty} \subset[0,1], \quad\left\{r_{n}\right\}_{n=0}^{\infty} \in[a, \infty)$ for some $a>0$ and $\xi_{n}=\sup _{u \in \mathbb{F}}\left(l_{n}-1\right) \phi\left(u, x_{n}\right)$. If $\sum_{i=0}^{\infty} \alpha_{n, i}=1, \forall_{n} \geq 0$ and $\lim _{\inf _{n \rightarrow \infty}} \alpha_{n, 0} \alpha_{n, i}>0, \forall i \geq 1$, then $x_{n} \rightarrow \Pi_{\mathbb{F} \cap \mathbb{G}} x_{0}$. $\square$

Furthermore, if $S_{i}=S, i \geq 1$ in Corollary 3.1, the following corollary can be obtained immediately.

Corollary 3.2. Suppose that, besides (D1),

$\left(\mathrm{E}_{1}\right) S: C \rightarrow C$ is closed and quasi- $\varphi$-asymptotically nonexpansive with $\left\{l_{n}\right\} \subset[1, \infty)$, $l_{n} \rightarrow 1$

$\left(\mathrm{E}_{2}\right) \mathrm{S}$ is either locally uniformly Lipschitz continuous or uniformly Hölder continuous with order $\Theta(\Theta>0), F(S)$ is bounded in $C$ and $F(S) \cap G \neq \varnothing$. Take the sequence $\left\{x_{n}\right\}_{n=1}^{\infty}$ generated by

$$
\left\{\begin{aligned}
x_{0} \in C, H_{0}=W_{0}=C, & \\
y_{n}=J^{-1}\left(\alpha_{n} J x_{n}+\left(1-\alpha_{n}\right) J S^{n} x_{n}\right), & \\
u_{n} \in C \text { such that } & \quad \quad\left(u_{n}, y\right)+\left\langle y-u_{n}, A u_{n}\right\rangle+\frac{1}{r_{n}}\left\langle y-u_{n}, J u_{n}-J y_{n}\right\} \geq 0, \quad \forall y \in C, \\
& \quad, \\
H_{n+1}= & \left\{v \in H_{n}: \phi\left(v, u_{n}\right) \leq \phi\left(v, x_{n}\right)+\xi_{n}\right\}, \\
W_{n+1}= & \left\{z \in W_{n}:\left\langle x_{n}-z, J x_{0}-J x_{n}\right\rangle \geq 0\right\}, \\
x_{n+1}= & \Pi_{H_{n+1} \cap W_{n+1} x_{0}, \quad \forall n \geq 0,}
\end{aligned}\right.
$$

where $\left\{\alpha_{n}\right\}_{n=0}^{\infty} \subset(0,1),\left\{r_{n}\right\}_{n=0}^{\infty} \in[a, \infty)$ for some $a>0$ and $\xi=\sup _{u \in F(S)}\left(l_{n}-1\right) \varphi\left(u, x_{n}\right)$ . If $\lim \inf _{n \rightarrow \infty} \alpha_{n}\left(1-\alpha_{n}\right)>0$, then $x_{n} \rightarrow \Pi_{F(S) \cap G} x_{0}$.

Author details

${ }^{1}$ College of Applied Science, Beijing University of Technology, Beijing 100124, PR China ${ }^{2}$ College of Mathematics, Jilin Normal University, Siping, Jilin 136000, PR China

Authors' contributions

All the authors read and approved the final manuscript.

Competing interests

The authors declare that they have no completing interests. 
References

1. Zhang F, Su YF: A general iterative method of fixed points for equilibrium problems and optimization problems. $J$ Syst Sci Complex 2009, 22:503-517.

2. Ceng LC, Al-Homidan S, Ansari QH, Yao JC: An iterative scheme for equilibrium problems and fixed point problems of strict pseudo-contraction mappings. J Comput Appl Math 2009, 223:967-974.

3. Zhang SS, Rao RF, Huang JL: Strong convergence theorem for a generalized equilibrium problem and a $k$-strict pseudocontraction in Hilbert spaces. Appl Math Mech, English 2009, 30(6):685-694.

4. Peng JW, Yao JC: Strong convergence theorems of iterative scheme based on the extragradient method for mixed equilibrium problems and fixed point problems. Math Comput Model 2009, 49:1816-1828.

5. Peng JW, Yao JC: A viscosity approximation scheme for system of equilibrium problems, nonexpansive mappings and monotone mappings. Nonlinear Anal Theory Methods Appl 2009, 71:6001-6010.

6. Cianciaruso F, Marino G, Muglia L: Iterative methods for equilibrium and fixed point problems for nonexpansive semigroups in Hilbert spaces. J Optim Theory App/ 2010, 146:491-509.

7. Qin XL, Chang SS, Cho YJ: Iterative methods for generalized equilibrium problems and fixed point problems with applications. Nonlinear Anal Real World Appl 2010, 11:2963-2972.

8. Takahashi W, Zembayashi K: Strong convergence theorem by a new hybrid method for equilibrium problems and relatively nonexpansive mappings. Fixed Point Theory App/ 2008, 2008:11, (Article ID 528476).

9. Takahashi W, Zembayashi K: Strong and weak convergence theorems for equilibrium problems and relatively nonexpansive mappings in Banach spaces. Nonlinear Anal Theory Methods Appl 2009, 70(1):45-57.

10. Chang SS, Lee HWJ, Chan CK: A new hybrid method for solving a generalized equilibrium problem, solving a variational inequality problem and obtaining common fixed points in Banach spaces, with applications. Nonlinear Anal Theory Methods Appl 2010, 73:2260-2270.

11. Chang SS, Kim JK, Wang XR: Modified block iterative algorithm for solving convex feasibility problems in Banach spacesm. J Inequal Appl 2010, 2010:14, (Article ID 869684).

12. Cioranescu I: Geometry of Banach spaces, Duality Mappings and Nonlinear Problems. In Mathematics and Its Applications. Volume 62. Edited by: Hazewinkel M. Kluwer Academic Publishers, Dordecht; 1990.

13. Alber Yl: Metric and generalized projection operators in Banach spaces: properities and applications. In Theory and Applications of Nonlinear operators of Accretive and Monotone Type, Lecture Notes in Pure and Applied Mathematics. Volume 178. Edited by: Kartosator AG. Marcel Dekker, New York; 1996:15-50.

14. Kamimura S, Takahashi W: Strong convergence of a proxiaml-type algorithm in a Banach space. SIAM J Optim 2002, 13(3):938-945.

15. Kohsaka F, Takahashi W: Existence and approximation of fixed points of firmly nonexpansive-type mappings in Banach spaces. SIAM J Optim 2008, 19(2):824-835.

16. Zhou HY, Gao GL, Tan B: Convergence theorems of a modified hybrid algorithm for a family of quasi- $\nabla-$ asymptotically nonexpansive mappings. J Appl Math Comput 2010, 32:453-464.

17. Matsushita S, Takahashi W: Weak and strong convergence theorems for relatively nonexpansive mappings in Banach spaces. Fixed Point Theory Appl 2004, 2004(1):37-47.

18. Blum E, Oettli W: From optimization and variational inequalities and equilibrium problems. Math Student 1994 63:123-145.

doi:10.1186/1687-1812-2011-17

Cite this article as: $\mathrm{Qu}$ and Cheng: A strong convergence theorem on solving common solutions for generalized equilibrium problems and fixed-point problems in Banach space. Fixed Point Theory and Applications 2011 2011:17.

\section{Submit your manuscript to a SpringerOpen ${ }^{\odot}$ journal and benefit from:}

Convenient online submission

- Rigorous peer review

- Immediate publication on acceptance

- Open access: articles freely available online

- High visibility within the field

- Retaining the copyright to your article

Submit your next manuscript at $\mathbf{s p r i n g e r o p e n . c o m ~}$ 